\title{
RATIOS OF DUAL GENERIC DEGREES OF A FINITE COXETER GROUP
}

\author{
DEAN ALVIS ${ }^{1}$
}

\begin{abstract}
A formula is obtained for the ratio of dual generic degrees of a finite Coxeter group.
\end{abstract}

1. Introduction. The purpose of this paper is to determine the ratio of the generic degrees corresponding to dual irreducible characters of a finite Coxeter group. These ratios could, of course, be found on a case by case basis, since the generic degrees are now known (see $[2, \mathbf{1}]$ ). We obtain a uniform formula for all cases (Corollary 2 below), which expresses the ratio in terms of the character table of the Coxeter group .

The present investigation started while the author was working with G. Lusztig on the generic degrees for type $H_{4}[\mathbf{1}]$. See $\S 4$ for a general result related to such calculations. The proof of Theorem 1 below was inspired by the proof of Lemma 1.11 of Lusztig's paper [9], which establishes the one variable case. The present methods apply to generic degrees in several variables.

The author wishes to thank the referee for several helpful comments.

2. A preliminary result. Throughout this paper $(W, S)$ is a finite Coxeter system, with set of distinguished generators $S$ and length function $l: W \rightarrow \mathbf{N}$. Let $R$ be the set of $W$-conjugates of elements of $S$, and let $\left\{u_{C}\right\}$ be a set of indeterminates, one for each conjugacy class $C \subseteq R$. Define $u_{s}=u_{C}$ if $s \in S \cap C$. Let $K$ be a field containing $\mathbf{Q}\left(\left\{u_{C}\right\}\right)$. The generic ring $A$ of $(W, S)$ over $K$ is the $K$-algebra with basis elements $T_{w}, w \in W$, and multiplication determined by

$$
\begin{array}{ll}
T_{s}^{2}=u_{s} T_{1}+\left(u_{s}-1\right) T_{s} & \text { if } s \in S, \\
T_{w} T_{w^{\prime}}=T_{w w^{\prime}} & \text { if } l(w)+l\left(w^{\prime}\right)=l\left(w w^{\prime}\right) .
\end{array}
$$

It is known that $A$ is a separable $K$-algebra with unit element $T_{1}$.

Let $s(1) \cdots s(l), l=l(w)$, be a reduced expression for $w \in W$. Define $\varepsilon_{w}=$ $\operatorname{sign}(w)=(-1)^{l}, u_{w}=u_{s(1)} \cdots u_{s(l)}$, and $\hat{T}_{w}=\varepsilon_{w} u_{w} T_{w^{-1}}^{-1}$. Then

$$
\hat{T}_{w}=\varepsilon_{w}\left(T_{s(1)}-u_{s(1)}+1\right) \cdots\left(T_{s(l)}-u_{s(l)}+1\right)
$$

by (1). The $K$-linear extension $a \rightarrow \hat{a}$ of $T_{w} \rightarrow \hat{T}_{w}$ is an involutory automorphism of $A$ (see $[6])$.

If $a, b \in A$ and $a b=\sum_{w} \alpha_{w} T_{w}, \alpha_{w} \in K$, set $B(a, b)=\alpha_{1}$. It is known that $B$ is a symmetric, associative nondegenerate $K$-bilinear form on $A$, with dual bases

Received by the editors July 20, 1983. Presented to the Society at the Bryn Mawr meeting, March 1982.

1980 Mathematics Subject Classification. Primary $20 \mathrm{C} 15$.

${ }^{1}$ Preparation supported in part by National Science Foundation Grant MCS-8300866. 
$\left\{T_{w}\right\},\left\{u_{w}^{-1} T_{w^{-1}}\right\}[4,5.12]$. Following $[7]$ we write

$$
T_{y^{-1}}^{-1}=\sum_{x \in W} u_{x}^{-1} \overline{R_{x, y}} T_{x}
$$

for $\overline{R_{x, y}} \in K$. Note $\overline{R_{x, y}}=B\left(T_{x^{-1}}, T_{y^{-1}}^{-1}\right)$.

Let $w_{0}$ be the longest element of $W$. If $x \in W$, then $l(x)+l\left(x^{-1} w_{0}\right)=l\left(w_{0}\right)$. Therefore

$$
\varepsilon_{x} \varepsilon_{x^{-1} w_{0}}=\varepsilon_{w_{0}}, \quad u_{x} u_{x^{-1} w_{0}}=u_{w_{0}}, \quad \text { and } \quad T_{x} T_{x^{-1} w_{0}}=T_{w_{0}}
$$

We require the following result, which is similar to [7, 2.1(iv)].

LEMMA 1. $\overline{R_{w_{0} y, w_{0} x}}=\overline{R_{x^{-1}, y^{-1}}}$.

PROOF. We have $\overline{R_{w_{0} y, w_{0} x}}=B\left(T_{y^{-1} w_{0}}, T_{x^{-1} w_{0}}^{-1}\right)$. Since

$$
\begin{gathered}
T_{y^{-1} w_{0}}=T_{y}^{-1} T_{w_{0}} \text { and } T_{x^{-1} w_{0}}^{-1}=T_{w_{0}}^{-1} T_{x}, \\
B\left(T_{y^{-1} w_{0}}, T_{x^{-1} w_{0}}^{-1}\right)=B\left(T_{y}^{-1}, T_{x}\right) .
\end{gathered}
$$

As $B\left(T_{y}^{-1}, T_{x}\right)=\overline{R_{x^{-1}, y^{-1}}}$, the proof is complete.

3. The main results. In this section we assume that $K$ is a splitting field for $A$. Let $V$ be a simple $A$-module, with representation $\rho: A \rightarrow \operatorname{End}_{K}(V)$ and character $\chi \in \operatorname{Irr} A$. The generic degree $d_{\chi}$ is defined by

$$
d_{\chi}=\frac{\operatorname{dim} V \cdot P}{\sum_{w \in W} u_{w}^{-1} \chi\left(T_{w}\right) \chi\left(T_{w^{-1}}\right)},
$$

where $P=\sum_{w} u_{w}$ is the Poincaré polyomial of $(W, S)[\mathbf{3}]$. The primitive central idempotent of $A$ corresponding to $\chi$ is

$$
e_{\chi}=\frac{d_{\chi}}{P} \sum_{w \in W} u_{w}^{-1} \chi\left(T_{w^{-1}}\right) T_{w}
$$

[5, 9.17]. Define an endomorphism $E(\rho)$ of $V$ by

$$
E(\rho)=\sum_{w \in W} u_{w}^{-1} \chi\left(T_{w^{-1}}\right) \rho\left(T_{w^{-1} w_{0}}^{-1}\right) .
$$

The dual $\hat{\rho}$ of $\rho$ is given by $\hat{\rho}(a)=\rho(\hat{a}), a \in A$. Note that $\hat{\rho}$ is an irreducible representation of $A$ with character $\hat{\chi}(a)=\chi(\hat{a})$. If $\chi$ corresponds to $\chi_{0} \in \operatorname{Irr} W$ under a specialization $u_{s} \rightarrow 1$ (see $[4,7.1]$ ), then $\hat{\chi}$ corresponds to sign $\otimes \chi_{0}$ under the same specialization by (2). Let $E(\hat{\rho})$ be defined as in (4), with $\hat{\rho}, \hat{\chi}$ replacing $\rho, \chi$.

LEMMA 2. $E(\hat{\rho})=\varepsilon_{w_{0}} E(\rho)$.

PROOF. Since $\left(T_{y^{-1} w_{0}}^{-1}\right)^{\uparrow}=\varepsilon_{w_{0} y} u_{w_{0} y}^{-1} T_{w_{0} y}$, we have

$$
\begin{aligned}
E(\hat{\rho}) & =\sum_{y \in W} u_{y}^{-1} \chi\left(\varepsilon_{y} u_{y} T_{y}^{-1}\right) \rho\left(\varepsilon_{w_{0} y} u_{w_{0} y}^{-1} T_{w_{0} y}\right) \\
& =\varepsilon_{w_{0}} \sum_{x, y \in W} u_{x}^{-1} \overline{R_{x^{-1}, y^{-1}}} \chi\left(T_{x^{-1}}\right) \rho\left(u_{w_{0} y}^{-1} T_{w_{0} y}\right) .
\end{aligned}
$$


The proof is completed by applying Lemma 1 and the identity

$$
\sum_{y \in W} u_{w_{0} y}^{-1} \overline{R_{w_{0} y, w_{0} x}} T_{w_{0} y}=T_{x^{-1} w_{0}}^{-1} .
$$

THEOREM 1. Suppose $V$ is a simple A-module with character $\chi$. Then $T_{w_{0}}^{2}$ acts on $V$ as the scalar $u_{w_{0}}\left(d_{\hat{\chi}} / d_{\chi}\right)$.

ProOF. Since $T_{w^{-1} w_{0}}^{-1}=T_{w_{0}}^{-1} T_{w}$ and $\rho\left(a e_{\chi}\right)=\rho(a), a \in A$, we have $E(\rho)=$ $\left(P / d_{\chi}\right) \rho\left(T_{w_{0}}^{-1}\right)$ by $(3)$ and (4). Similarly, $E(\hat{\rho})=\left(P / d_{\hat{\chi}}\right) \hat{\rho}\left(T_{w_{0}}^{-1}\right)$. Also, $\hat{\rho}\left(T_{w_{0}}^{-1}\right)=$ $\varepsilon_{w_{0}} u_{w_{0}}^{-1} \rho\left(T_{w_{0}}\right)$. The theorem now follows from Lemma 2 .

Corollary 1 (Kilmoyer [8, Proposition 14(ii)]). $T_{w_{0}}^{2}$ is central in $A$.

For $C$ a conjugacy class in $R$, define

$$
e(\chi, C)=|C| \chi_{0}(s) / \operatorname{dim} V, \quad s \in S \cap C,
$$

where $\chi_{0}(s)=\chi\left(T_{s}\right)_{u_{s}=1}$. Then $e(\chi, C)$ is a rational integer $[\mathbf{5}, 9.31]$. Let $w_{0}=$ $s_{1} s_{2} \cdots s_{N}, s_{i} \in S$, be a reduced expression for $w_{0}$. Then $T_{w_{0}}=T_{s_{1}} \cdots T_{s_{N}}$. It is known that the number of $s_{i}(1 \leq i \leq N)$ in a given class $C \subseteq R$ is exactly $|C|$. Arguing as in [3], we see that the multiplicity of $u_{s}$ as an eigenvalue of $\rho\left(T_{s}\right)$ is $\left(\chi_{0}(s)+\chi_{0}(1)\right) / 2$. Therefore

$$
\operatorname{det} \rho\left(T_{w_{0}}^{2}\right)=\left(u_{w_{0}} \prod_{C} u_{C}^{e(\chi, C)}\right)^{\operatorname{dim} V}
$$

Thus $T_{w_{0}}^{2}$ acts on $V$ as a scalar of the form $\lambda u_{w_{0}} \prod_{C} u_{C}^{e(\chi, C)}$, with $\lambda$ a root of unity. This scalar must assume the value 1 when each indeterminate $u_{C}$ is replaced by 1 since $w_{0}^{2}=1$, and therefore $\lambda=1$. Hence we have $\rho\left(T_{w_{0}}^{2}\right)=u_{w_{0}} \prod_{C} u_{C}^{e(\chi, C)}$, a formula first observed by Springer (see [3]). This, together with the theorem, proves the following.

COROLlaRY 2. $d_{\hat{\chi}} / d_{\chi}=\prod_{C} u_{C}^{e(\chi, C)}$, the product taken over all conjugacy classes $C \subseteq R$.

4. An application. In this section we derive a recursion formula giving certain of the generic degrees for $W$ in terms of those for the standard parabolic subgroups $W_{J}=\langle J\rangle, J \subseteq S, J \neq S$. A key ingredient in the formula is the ratio $d_{\hat{\chi}} / d_{\chi}$, which may be found using the character table for $W$ (Corollary 2).

Let $A_{J}$ be the subalgebra of $A$ spanned by the $T_{w}$ for $w \in W_{J}$. We assume $K$ is a splitting field for all $A_{J}, J \subseteq S$. If $\chi \in \operatorname{Irr} A$ and $\theta \in \operatorname{Irr} A_{J}$, let $m(\chi, \theta)$ be the multiplicity of $\theta$ in the restriction $\operatorname{Res}_{A_{J}}^{A}(\chi)$ of $\chi$ to $A_{J}$ (or, equivalently, of $\theta_{0}$ in $\left.\operatorname{Res}_{W_{J}}^{W}\left(\chi_{0}\right)\right)$. We have

$$
\frac{P}{P_{J}} d_{\theta}=\sum_{\chi \in \operatorname{Irr} A} m(\chi, \theta) d_{\chi}
$$

where $P_{J}$ is the Poincaré polynomial for $\left(W_{J}, J\right)$. This is established by Surowski in [10] for the crystallographic cases. In general, (5) may be easily proved using 
the fact that the generic degrees $d_{\chi}$ are the unique solution to the following linear system (see [1]).

$$
\sum_{\chi} \chi\left(T_{w}\right) d_{\chi}= \begin{cases}P & \text { if } w=1 \\ 0 & \text { if } w \neq 1\end{cases}
$$

We also have

$$
\sum_{\substack{J \subseteq S \\ \theta \in \operatorname{Irr} A_{J}}}(-1)^{|J|} m(\chi, \theta) m\left(\chi^{\prime}, \theta\right)=\delta_{\hat{\chi}, \chi^{\prime}}
$$

for $\chi, \chi^{\prime} \in \operatorname{Irr} A$. This corresponds, on the level of $W$, to the fact that

$$
\sum_{J \subseteq S}(-1)^{|J|} \operatorname{Ind}_{W_{J}}^{W} \operatorname{Res}_{W_{J}}^{W}\left(\chi_{0}\right)=\operatorname{sign} \bigotimes \chi_{0}
$$

for $\chi_{0} \in \operatorname{Irr} W$. Combining (5) and (6), we obtain

$$
d_{\hat{\chi}}=\sum_{\substack{J \subseteq S \\ \theta \in \operatorname{Irr} A_{J}}}(-1)^{|J|} \frac{P}{P_{J}} m(\chi, \theta) d_{\theta} .
$$

Hence

$$
\left(\frac{d_{\hat{\chi}}}{d_{\chi}}-(-1)^{|S|}\right) d_{\chi}=\sum_{\substack{J \neq S \\ \theta \in \operatorname{Irr} A_{J}}}(-1)^{|J|} \frac{P}{P_{J}} m(\chi, \theta) d_{\theta} .
$$

If $|S|$ is odd or $d_{\hat{\chi}} \neq d_{\chi}$, we may divide by $\left(d_{\hat{\chi}} / d_{\chi}\right)-(-1)^{|S|}$ to solve $(7)$ for $d_{\chi}$. This is the desired (partial) recursion formula.

In practice, a slightly different technique has been used. Using (5) and transitivity of induction, the right-hand side of (7) may be replaced by a sum

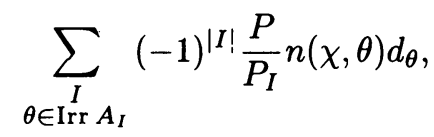

where $I$ ranges over the maximal subsets of $S$. The coefficients $n(\chi, \theta)$ are certain integers, and are not unique. They may be found by solving the underdetermined linear system

$$
\hat{\chi}-(-1)^{|S|} \chi=\sum_{\substack{I \\ \theta \in \operatorname{Irr} A_{K}}}(-1)^{|I|} n(\chi, \theta) \operatorname{Ind}_{A_{I}}^{A}(\theta)
$$

(see, for example, $[2])$.

\section{REFERENCES}

1. D. Alvis and G. Lusztig, The representations and generic degrees of the Hecke algebra of type $H_{4}, \mathrm{~J}$. Reine Angew. Math. 336 (1982), 201-212.

2. C. T. Benson, The generic degrees of the irreducible characters of $E_{8}$, Comm. Algebra 7 (1979), 1199-1209.

3. C. T. Benson and C. W. Curtis, On the degrees and rationality of certain characters of finite Chevalley groups, Trans. Amer. Math. Soc. 165 (1972), 251-273; ibid. 202 (1975), 405-406.

4. C. W. Curtis, N. Iwahori and R. Kilmoyer, Hecke algebras and characters of parabolic type of finite groups with $(B, N)$-pairs, Inst. Hautes Études Sci. Publ. Math. 40 (1971), 81-116. 
5. C. W. Curtis and I. Reiner, Methods of representation theory, Vol. I, Wiley-Interscience, New York, 1981.

6. N. Iwahori, On the structure of the Hecke ring of a Chevalley group over a finite field, J. Fac. Sci. Tokyo Univ. (2) 10 (1964), 215-236.

7. D. Kazhdan and G. Lusztig, Representations of Coxeter groups and Hecke algebras, Invent. Math. 53 (1979), 165-184.

8. R. Kilmoyer, Some irreducible complex representations of a finite group with BN pair, Ph.D. Dissertation, M.I.T., 1969.

9. G. Lusztig, Unipotent characters of the symplectic and odd orthogonal groups over a finite field, Invent. Math. 64 (1981), 263-296.

10. D. B. Surowski, Degrees of irreducible characters of $(B, N)$-pairs of types $E_{6}$ and $E_{7}$, Trans. Amer. Math. Soc. 243 (1978), 235-249.

Department of Mathematics, University of Notre Dame, Notre Dame, IndiANA 46556 